\title{
INDICES OF MAXIMAL SUBGROUPS OF INFINITE SYMMETRIC GROUPS
}

\author{
RALPH W. BALL
}

Let $M$ be an infinite set with cardinal number $|M|=X, S(X, Y)$ $=\{s: s$ is a permutation of $M$ with $\mid$ spt $s \mid<Y\}$, where spt $s$ $=\{m \in M: s(m) \neq m\}$. If $X$ is a cardinal number, denote its successor by $X^{+}$; the smallest infinite cardinal shall be denoted by $d$. Higman [3] has shown that $S(X, d)$ has only one proper subgroup of index less than $X$, the alternating subgroup $A(X)$, and $A(X)$ has no proper subgroups of index less than $X$. Gaughan [2] has extended these results showing that if $d<Y \leqq X^{+}$, then $S(X, Y)$ has no proper subgroups of index less than $X$.

One might conjecture that any proper maximal subgroup of $S(X, Y)$ would have minimal index, namely $X$. It is the purpose of this paper to demonstrate that such is not the case by constructing examples with index greater than $X$. It will also be shown that all intransitive proper maximal subgroups of $S(X, Y)$ do have index $X$.

Hereafter, maximal shall mean proper maximal. Let $M$ be partitioned in to $P$ and $Q$ with $|M|=X \geqq d,|P|=X,|Q|=Z, 0<Z \leqq X$, $d \leqq Y \leqq X^{+}$. Let $J(Z)=S(Q) \cdot S(P), J(Y, Z)=J(Z) \cap S(X, Y)$. It has been shown [1] that if $Z<d$, then $J(Y, Z)$ is a maximal subgroup of $S(X, Y)$. In fact every intransitive maximal subgroup of $S(X, Y)$ is of this form. Let $s \in S\left(X, X^{+}\right)$, and define $P_{s}=\{x \in P: s(x) \in Q\}$ and $Q_{s}=\{y \in Q: s(y) \in P\}$. For each $s \in S\left(X, X^{+}\right)$define the transfer index of $s=T(s)=\max \left\{\left|P_{s}\right|,\left|Q_{s}\right|\right\}$. Now define $L(Y, Z)$ as follows: If $d \leqq Z<Y \leqq X^{+}, Z<X$, then $L(Y, Z)=\{s \in S(X, Y): T(s)<Z\}$. If $d<Y \leqq Z \leqq X$ and $Y$ has an immediate predecessor $Y^{-}$, then $L(Y, Z)$ $=\left\{s \in S(X, Y): T(s)<Y^{-}\right\}$. It has been shown [1] that $L(Y, Z)$ is a (transitive) maximal subgroup of $S(X, Y)$.

THEOREM 1. Let $H$ be an intransitive maximal subgroup of $S(X, Y)$. Then $[S(X, Y): H]=X$.

Proof. By [1], $H=J(Y, Z)$ with $0<Z<d$. By Gaughan [2], $[S(X, Y): H] \geqq X$. Since $H$ is intransitive, $A(X) \Phi H$, so by maximality, $S(X, Y)=A(X) \cdot H$, and $[S(X, Y): H] \leqq|A(X)|=X$.

Let $H$ be any subgroup of a group $G$. By $\mathrm{Cl}(H)$ is meant the set of all conjugates of $H$ in $G, \mathrm{Cl}(H)=\left\{g \mathrm{Hg}^{-1}: g \in G\right\}$. It is well known that $[G: N(H)]=|\mathrm{Cl}(H)|$, where $N(H)$ is the normalizer of $H$ in $G$.

Received by the editors October 1, 1965 and, in revised form, April 16, 1967. 
A suitable choice of cardinals $X, Y$, and $Z$ will now be made to construct $H=L(Y, Z)$ with $[S(X, Y): H]>X$.

Lemma 1. Let $d \leqq Z \leqq X$. Let $C$ be a class of subsets of $M$ of cardinality $Z$ such that if $Q_{1}, Q_{2} \in C, Q_{1} \neq Q_{2}$, then $\left|Q_{1}-Q_{2}\right|=Z$. Then $|C| \leqq X^{Z}$, and there is such a class $C_{0}$ such that $\left|C_{0}\right|=X^{Z}$.

Proof. The set of all subsets of $M$ of cardinality $Z$ has cardinality $X^{z}$. Thus $|C| \leqq X^{z}$. Let $M=\bigcup_{x \in M} Q_{x}$ disjointly such that for all $x \in M,\left|Q_{x}\right|=Z$. Also let $M=\cup_{a \in A} P_{a}$ disjointly such that $|A|=Z$ and for all $a \in A,\left|P_{a}\right|=X$. Let $F$ be the family of nonvoid subsets of $M$ such that for each $D \in F$ and for each $a \in A,\left|D \cap P_{a}\right|=1$. $F$ has the property that $D_{1} \Phi D_{2}$ if $D_{1} \neq D_{2}$. Furthermore $|F|=X^{z}$, and $|D|=Z$ if $D \in F$. Let $E_{D}=\cup_{x \in D} Q_{x}$, and $C_{0}=\left\{E_{D}: D \in F\right\}$. Now $C_{0}$ has the desired property, and $E_{D_{1}}=E_{D_{2}}$ if and only if $D_{1}=D_{2}$, so $\left|C_{0}\right|=|F|=X^{Z}$.

THEOREM 2. Let $M$ be partitioned into $P$ and $Q$ as before with $d<Z$ $<Y \leqq X^{+}, Z<X$. Then $[S(X, Y): L(Y, Z)]=X^{Z}$.

Proof. By [1], $L(Y, Z)=J(Y, Z) \cdot S(X, Z)$. Hence for any $s \in S(X, Y), s L(Y, Z) s^{-1}=s[J(Y, Z) \cdot S(X, Z)] s^{-1}=\left[s J(Y, Z) s^{-1}\right]$ $\cdot S(X, Z)=[S(s(Q), Y) \cdot S(s(P), Y)] \cdot S(X, Z)$. Thus $s L(Y, Z) s^{-1}$ $\neq r L(Y, Z) r^{-1}$ if and only if $|s(Q)-r(Q)|=Z$ and $|r(Q)-s(Q)|=Z$. By Lemma 1, there are $X^{Z}$ subsets of $M$ of cardinality $Z$ having this property and at most $X^{z}$ such subsets. Since $Z<Y$, there are $X^{Z}$ permutations in $S(X, Y)$ mapping $Q$ onto distinct members of this class of subsets of $M$, hence $|\mathrm{Cl}(L(Y, Z))|=X^{Z}$. By the maximality and nonnormality of $L(Y, Z), N(L(Y, Z))=L(Y, Z)$. Thus $[S(X, Y): L(Y, Z)]=|\mathrm{Cl}(L(Y, Z))|=X^{Z}$.

Lemma 2. There are infinite cardinals $Z$ and $X$ such that $Z<X$ and $X<X^{z}$.

Proof. Let $Z$ be an infinite cardinal, $A$ a well-ordered set of cardinality $Z,\left\{Z_{a}\right\}_{a \in A}$ a family of cardinal numbers such that $Z_{a}>Z$ for each $a \in A$, and if $a, b \in A$ with $a<b$, then $Z_{a}<Z_{b}$. Let $f$ be a oneto-one function from $A$ into $A$ such that $a<f(a)$ for each $a \in A$. Then by the theorem of Koenig and Zermelo, $X=\sum_{a \in A} Z_{a}<\prod_{a \in A} Z_{f(A)}$ $\leqq X^{z}$.

THEOREM 3. There are cardinal numbers $X$ and $Y, Y \leqq X^{+}$, such that $S(X, Y)$ contains maximal subgroups with index larger than $X$.

Proof. By Lemma 2, choose $X, Y$, and $Z$ such that $Z<Y \leqq X^{+}$, $Z<X$, and $X<X^{z}$. By Theorem 2, $[S(X, Y): L(Y, Z)]=X^{z}>X$. 
Theorem 3 is somewhat limited by the choice of $X$ and $Z$. However, for $S\left(X, X^{+}\right)$, maximal subgroups of index greater than $X$ can be demonstrated for any choice of $X \geqq d$. Let $s \in S\left(X, X^{+}\right)$and define $P_{s}^{\prime}=P-P_{s}$ and $Q_{s}^{\prime}=Q-Q_{s}$. For each $s \in S\left(X, X^{+}\right)$define the $r e-$ mainder index of $s=R(s)=\max \left\{\left|P_{s}^{\prime}\right|,\left|Q_{s}^{\prime}\right|\right\}$. Now define $L\left(X^{+}, X\right)$ $=\left\{s \in S\left(X, X^{+}\right): T(s)<X\right.$ or $\left.R(s)<X\right\}$. It has been shown [1] that $L\left(X^{+}, X\right)$ is a (transitive) maximal subgroup of $S\left(X, X^{+}\right)$.

Theorem 4. For $X \geqq d,\left[S\left(X, X^{+}\right): L\left(X^{+}, X\right)\right]>X$.

Proof. Partition $P$ into $P_{1}$ and $P_{2}$ such that $\left|P_{1}\right|=\left|P_{2}\right|=X$. By Lemma 1 choose a collection $C$ of subsets of $P_{1}$ such that $|C|=X^{x}$ $=2^{x}$, and if $A, B \in C$ with $A \neq B$, then $|A-B|=X$. Similarly choose a collection $D$ of subsets of $Q$ with the same properties. Let $f$ be a one-to-one function from $C$ onto $D$. For each $A \in C$ choose a corresponding $s \in S\left(X, X^{+}\right)$such that $s(A)=f(A), \quad s(P-A)=P-A$, $s(f(A))=A, s(Q-f(A))=Q-f(A)$. Note that $T(s)=R(s)=X$, so $s \in L\left(X^{+}, X\right)$. Let $F$ denote the family of functions so defined. Let $s$ and $r \in F, s \neq r, s$ and $r$ correspond to $A$ and $B \in C$ respectively. Now $P_{r^{-1} s} \supseteq A-B$, so $X \geqq\left|P_{r^{-1}}\right| \geqq|A-B|=X$. Thus $T\left(r^{-1} s\right)=X$. Also $P_{r^{-1}}^{\prime} \supseteq(P-A)-B=P-(A \cup B) \supseteq P_{2}, \quad$ so $\quad X \geqq\left|P_{r^{-1} \varepsilon}^{\prime}\right| \geqq\left|P_{2}\right|=X$. Thus $R\left(r^{-1} s\right)=X$. Hence $r^{-1} s \notin L\left(X^{+}, X\right)$, so $r$ and $s$ determine different cosets of $L\left(X^{+}, X\right)$ in $S\left(X, X^{+}\right)$. Thus $2^{X}=\left|S\left(X, X^{+}\right)\right|$ $\geqq\left[S\left(X, X^{+}\right): L\left(X^{+}, X\right)\right] \geqq|F|=|C|=2^{X}$, so $\left[S\left(X, X^{+}\right): L\left(X^{+}, X\right)\right]$ $=2^{X}>X$.

\section{BiBLIOGRAPHY}

1. R. W. Ball, Maximal subgroups of symmetric groups, Trans Amer. Math. Soc. 121 (1966), 393-407.

2. E. D. Gaughan, The index problem for infinite symmetric groups, Proc. Amer. Math. Soc. 15 (1964), 527-528.

3. G. Higman, On infinite simple permutation groups, Publ. Math. Debrecen 3 (1954), 221-226.

4. Waclaw Sierpinski, Cardinal and ordinal numbers, PWN, Warsaw, 1958.

New Mexico Institute of Mining and Technology 